\title{
EVOLUÇÃO DAS UNIDADES MAGMÁTICAS DO COMPLEXO GRANITÓIDE ITAQUI (PRÉ-CAMBRIANO SUPERIOR), ESTADO DE SÃO PAULO, BRASIL: ASPECTOS GEOLÓGICOS, PETROGRÁFICOS E GEOQUÍMICOS (ELEMENTOS MAIORES)
}

\author{
EBERHARD WERNICK*, CLÁUDIO J. FERREIRA** e PAUL K. HÖRMANN***
}

\begin{abstract}
EVOLUTION OFIGNEOUS UNITS OF ITAOUI GRANITOID COMPLEX (LATE PRECAMBRIAN), STATE OF SÃO PAULO, BRAZIL: GEOLOGICAL, PETROGRAPHIC AND GEOCHEMICAL (MAJOR ELEMENTS) ASPECTS. Chemical data from the 10 magmatic units which build up the calcalkaline granodiorite/monzogranitic Itaqui complex (State of São Paulo, SE Brazil) are discussed. The late Precambrian granitoid is intrusive in the low grade metamorphic São Roque Group, but extensive contacts are done by the expressive Taxaquara transcurrent fault. The chemical data combined with mineralogical and textural ones as well as with the intrusion sequence of the magmatic units allowed the definition of two main magmatic cycles each subdivided in two subcycles. The cycles are separated by a phase of intrusion of melaquartzmonzonite dikes and the younger cycle is followed by the emplacement of tourmaline-muscovite monzogranitic aplites possibly related to the older cycle. Each cycle/subcycle hás its own magmatic evolution trend defined by several cationic diagrams and involving the fractionation of mafic (hornblende/biotite), plagioclase and accessory minerais by different mechanisms.
\end{abstract}

Keywords: Calc-alkaline granite, geochemical evolution, multicationic diagrams, magmatic cycles, Itaqui complex.

\begin{abstract}
RESUMO São discutidos dados químicos referentes às 10 unidades magmáticas que constituem o Complexo Cálcio-Alcalino Itaqui (SP, SE Brasil). O complexo associa-se à falha transcorrente de Taxaquara e sua arquitetura magmática resulta de quatro etapas de acresção magmática. Os dados químicos combinados com dados mineralógicos e texturais permitem a caracterização de dois ciclos magmáticos principais, cada um subdividido em dois subciclos. Os dois ciclos são separados temporalmente pela colocação de uma fase de diques de mela-quartzo monzonitos e são seguidos de turmalina-muscovita aplitos monzograníticos, possivelmente vinculados geneticamente ao primeiro ciclo magmático. Cada ciclo/subciclo apresenta evolução própria discutida em diferentes diagramas geoquímicos catiônicos e envolvem o fracionamento de minerais máficos, plagioclásio e acessórios.
\end{abstract}

Palavras-chave: Granito cálcio-alcalino, evolução geoquímica, diagramas multicatiônicos, ciclos magmáticos, complexo Itaqui.

INTRODUĊ̃̃O O Complexo Granitóide Cálcio-Alcalino Itaqui (Ferreira 1991) é composto por 10 unidades magmáticas cuja acresção ocorreu em quatro etapas intrusivas principais, vinculadas a diversas fases de removimentação da Falha Transcorrente de Taxaquara (Wernick \& Ferreira 1991). A caracterização da evolução deste magmatismo pelo exame conjunto de dados petrográficos e químicos (elementos maiores) é o objetivo do presente trabalho.

O COMPLEXO GRANITÓIDE ITAQUI Complexo Granitóide Itaqui, de idade pré-cambriana superior, situa-se nos arredores da cidade de São Paulo, Estado de São Paulo, Sudeste do Brasil. Apresenta forma de gota, cujo eixo maior tem direção WNW-ESE, e área de $120 \mathrm{~km}^{2}$. Sua porção norte é intrusiva nos metamorfltos de baixo grau do Grupo São Roque e seu contato sul é dado pela Falha Transcorrente Taxaquara (Fig. 1). A fase principal de intrusão ocorreu entre a segunda e a terceira das três fases de dobramento regional que afetaram as rochas encaixantes.

A arquitetura do complexo (Fig. 2) é dada por dez unidades magmáticas, listadas de acordo com sua sequência de colocação: 1. Granodiorito Barueri (BA); 2. Monzogranito Pedreira Cantareira (PC); 3. Monzogranito Torre (TO); 4. diques de mela-quartzo monzonitos porfiríticos (DMP) e $\mathbf{5}$. eqüigranulares (DME); 6. Quartzo Monzonito Cruz Preta
(CP); 7. Monzogranito Aldeia da Serra (AS); 8. Monzogranito Mutinga (MU); 9. Monzogranito Suru (SU); e 10. diques de turmalina-muscovita aplitos de monzogranitos (APL).

A arquitetura do Complexo Itaqui resulta de quatro fases de acresção. A ascenção magmática ocorre via propagação de diques ao longo de falhas e fraturas profundas que se associam às diferentes fases de movimentação da falha Taxaquara:

1. Fase de acresção principal por meio da colocação forçada de magmas em espaços gerados por movimentos pull apart, frutos da movimentação dextral da Falha Taxaquara. Resultam na colocação das Unidades Barueri (BA) e Pedreira Cantareira (PC). A intrusão forçada retrata-se na Unidade BA por intensas estruturas de fluxo, feições tipo schlieren, diques de megacristais etc.

2. Fase de acresção de espessos diques precoces (Unidades Torre, TO, diques máficos, DMP/DME e Cruz Preta, CP) em regime distensional.

3. Fase de acresção por esforços hidráulicos retratada pela colocação de stocks das Unidades Aldeia da Serra (AS) e Mutinga (MU).

4. Fase de acresção tardia, ainda em regime distensional com a colocação da Unidade Suru (SU), que constitui um fragmento de dique anelar marginal e dos turmalinamuscovita aplitos tardios (APL) (Fig. 2).

\footnotetext{
* Departamento de Petrologia e Metalogenia, Instituto de Geociências e Ciências Exatas, Universidade Estadual Paulista, Caixa Postal 178, CEP13506 970 , Rio Claro, SP, Brasil, Fax (0195)249644

** Instituto Geológico, Secretaria do Meio Ambiente, Avenida Miguel Stéfano, 3900, CEP 04301-903, São Paulo, SP, Fax (011) 2768572

*** Mineralogisch-Petrographisches Institut der Universitãt, Ludewig-Meyn Strasse 10, W - 24098 Kiel 1, Alemanha
} 

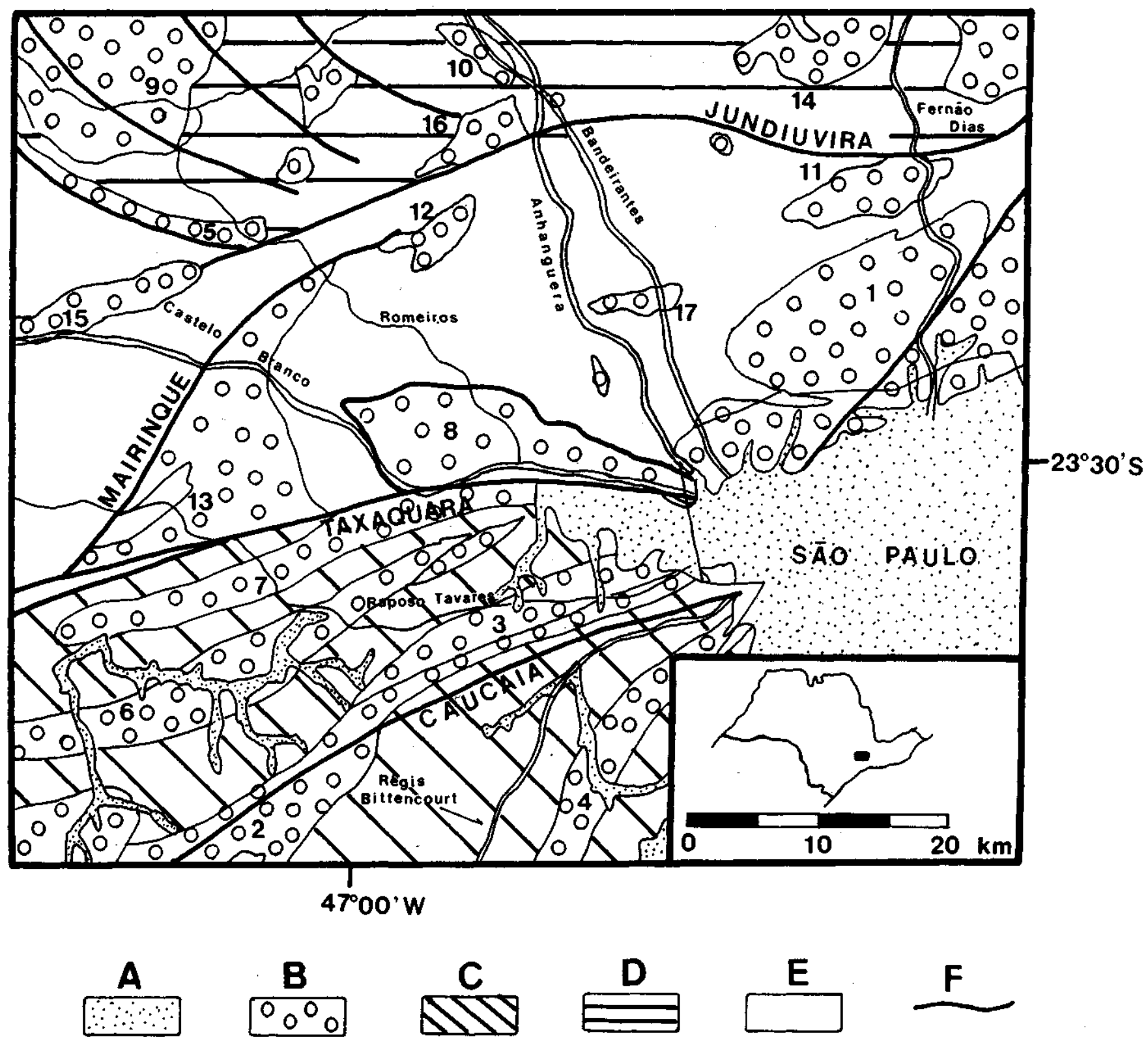

Figura 1 - Localização e situação geológica do Complexo Granitóide Itaqui. Convenções: A. sedimentos; B. granitóides; C. Complexo Embu; D. Complexo Amparo; E. Grupo São Roque; F. falhas transcorrentes. Compilado de Bistrichi et al (1981), Janasi et al. (1990) e Janasi \& Ulbrich (1992)

Figure 1 - Geological setting of Itaqui granitoid complex. Key: A. sediments; B. granitoids; C. Embu complex; D. Amparo complex; E. São Roque group; F. transcurrent faults. After Bistrichi et al. (1981), Janasi et al. (1990) and Janasi \& Ulbrich (1992)

DADOS QUÍMICOS Para a realização deste trabalho, os autores dispuseram de 48 análises químicas em fase de tratamento integrado (Ferreira, tese de doutoramento em andamento). Dessas análises, 26 foram tratadas sob aspecto de seus ETR em conexão com alguns elementos traços (Wernick et al 1991, 1992) e o total de amostras em conexão com sua tipologia de zircão (Ferreira et al. 1992). A listagem dos dados pode ser obtida por solicitação ao autor C. J. Ferreira.

DISCUSSÃO DOS DADOS A discussão dos dados será feita sob dois aspectos: a caracterização de ciclos magmáticos e a discussão de sua evolução magmática.

Ciclos magmáticos Neste trabalho, o conceito de ciclo magmático é tomado próximo ao de super unit de
Pitcher (1985). Um ciclo é integrado por um conjunto de unidades magmáticas consanguíneas que apresentam estreitas relações espaciais e temporais. Cada unidade (ou fácies) do ciclo é caracterizada por feições mineralógicas, texturais e químicas específicas e corresponde a um impulso magmático simples. A existência de vários ciclos magmáticos implica na geração de complexos múltiplos e compostos (Pitcher 1985).

Denomina-se de fase magmática a um conjunto de unidades cuja colocação ocorreu em condições tectônicas peculiares, retratada por tipos de jazimentos específicos (p. ex., a fase magmática de diques tardios). Um complexo múltiplo e composto desenvolve-se pela coalescência de sucessivos impulsos magmáticos reunidos em fases, que retratam a evolução tectônica do complexo, e em um ou mais ciclos magmáticos. 


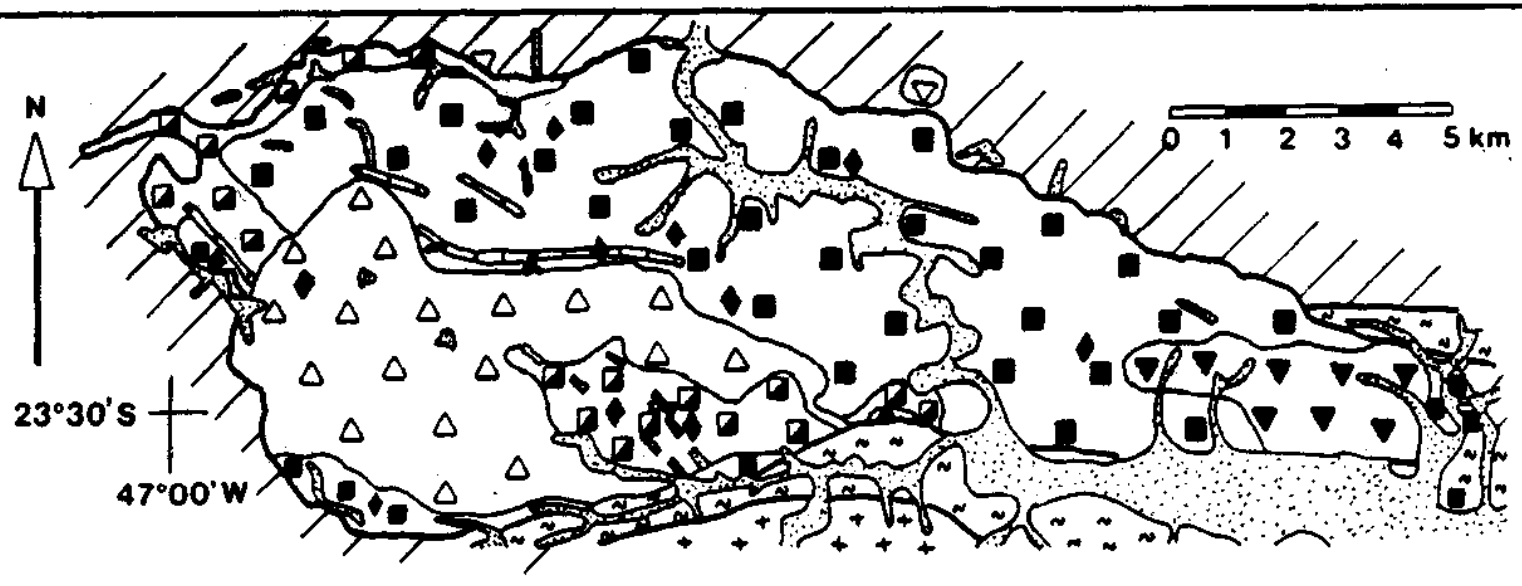

\begin{tabular}{|c|c|c|c|c|c|}
\hline \multicolumn{3}{|c|}{ COMPLEXO GRANITÓIDE ITAQUI } \\
\hline $\begin{array}{c}\text { FASE } \\
\text { INTRUSIVA }\end{array}$ & $\begin{array}{c}\text { UNIOADE } \\
\text { ÍGNEA }\end{array}$ & $\begin{array}{l}\text { ASSOCIACÃO } \\
\text { MAGMÁTICA }\end{array}$ & \multicolumn{2}{|c|}{ CICLO } \\
MAGMÁTICO
\end{tabular}

SEDIMENTOS

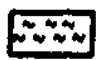

ZONAS DE CISALHAMENTO

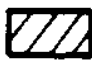

METAMORFITOS (GRUPO SÃo ROQUE)

GRANITO ITAPEVI

Figura 2 - Geologia do Complexo Granitóide Itaqui, SP. Modificado de Ferreira \& Wernick (1989), Ferreira (1991) e Wernick \& Ferreira (1991). Convenções: BA. Granodiorito Baruerí; PC. Monzogranito Pedreira Cantareira; TO. Monzogranito Torre; DMP. dique de quartzo monzonito máfico porfirítico; $\mathbf{D M E}$. dique de quartzo monzonito máfico equigranular; CP. Quartzo Monzonito Cruz Preta; AS. Monzogranito Aldeia da Serra; MU. Monzogranito Mutinga; Su. Monzogranito Suru; e APL. aplitos

Figure 2 - Geology of Itaqui granitoid complex, SP. After Ferreira \& Wemick (1989), Ferreira (1991) and Wernick \& Ferreira (1991). Key: BA. Barueri granodiorite; PC. Pedreira Cantareira monzogranite; TO. Torre monzogranite; DMP. quartz monzonite mafic porphyritic dike; DME. quartz monzonite mafic even-grained dike; CP. Cruz Preta quartz monzonite; AS. Aldeia da Serra monzogranite; MU. Mutinga monzogranite; SU. Suru monzogranite; and APL. aplite

Baseado nos elementos maiores, pode ser definida no Complexo Itaqui a presença de dois grandes ciclos magmátícos (ciclos $\mathrm{I} \mathrm{e} \mathrm{u}$ ), conforme a figura 4.0 ciclo I, mais antigo, compreende as unidades BA, PC e TO, e o ciclo ii as unidades $\mathrm{CP}, \mathrm{AS}, \mathrm{MU}$ e SU. Os dois ciclos são separados por uma fase de colocação de diques de mela-quartzo monzonitos (DMP e DME) e seguidos da fase de intrusão dos turmalina-muscovita aplitos (APL).

$\mathrm{O}$ ciclo I inicia-se com rochas porfiróides (BA e $\mathrm{PC}$ ) e termina com rochas equigranulares (TO) e compreende rochas com hornblenda e biotita (BA e PC) ou apenas portadoras de biotita (TO). A relação hornblenda/ biotita na Unidade Barueri é maior do que a da Unidade Pedreira Cantareira. Sob aspectos dos ETR, o ciclo I caracteriza-se por razões $\mathrm{La} / \mathrm{Lu}$ crescentes com a sequência de colocação de suas unidades magmáticas (Wernick et al. 1992).

O ciclo II inicia-se com rochas porfiríticas de matriz fina a média (unidade CP), passando para rochas porfiríticas seriais e inequigranulares de granulação média (AS e MU) e termina com rochas equigranulares (SU).Todas as unidades contém apenas biotita como mineral fềmico. Em termos de ETR, o ciclo II caracteriza-se por razões $\mathrm{La} / \mathrm{Lu}$ decrescentes com a sequência de colocação de suas unidades magmáticas (Wernick et al. 1992).

A variação composicional das diversas unidades dos dois ciclos é mais ou menos ampla. Enquanto uma variação composicional mais ampla pode ser atribuída a diferenciações in situ, após a colocação do magma ou do mush magmático, uma variacão mais restrita sugere que uma câmara magmática estratificada é drenada nos seus diferentes níveis diferenciados. O predomínio de unidades com grande variação composicional no Complexo Itaqui (Fig. 4) sugere a operação de mecanismos de diferenciação in situ que agiram sobre impulsos magmáticos sucessivos. Estes impulsos são provenientes de uma fonte magmática com pelo menos dois estágios evolutivos com características bastante distintas (ciclos I e II). 

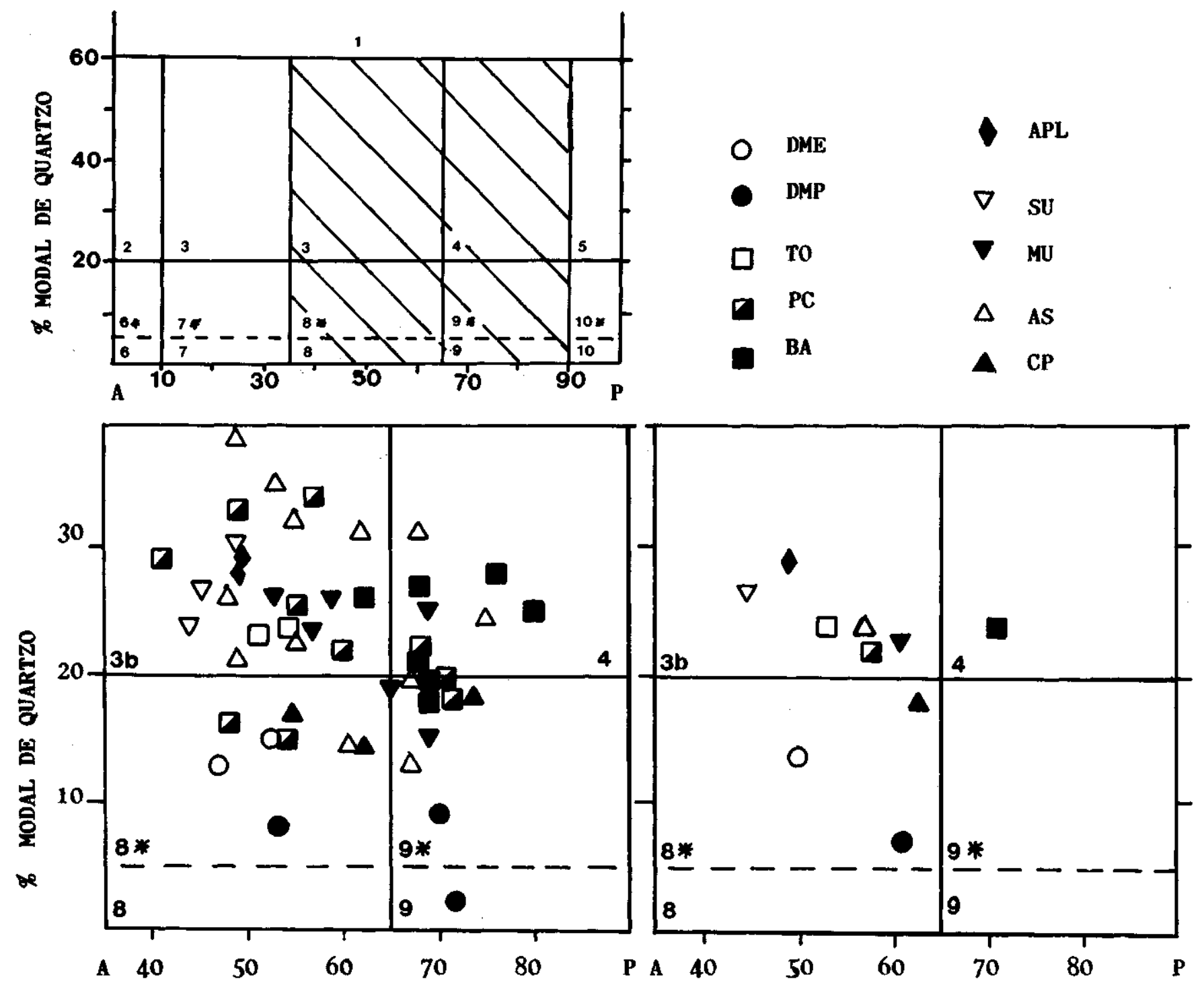

Figura 3 - Classificação modal no diagrama QAP retangular (Philpotts, 1990) das unidades ígneas do Complexo Granitóide Itaqui. Dados totais à esquerda e dados médios por unidade à direita. Símbolos segundo a figura 2. Segundo Ferreira (1991) e Ferreira \& Wernick (1991)

Figure 3 - Modal QAP classification (after Philpotts, 1990) of igneous units of the Itaqui Complex. Ali data on the left, and averages on the right. Key as Figure 2. After Ferreira (1991) and Ferreira \& Wernick (1991)

Dados de tipologia de zircão (Ferreira et al. 1992) indicam que as temperaturas de cristalização do ciclo I são menores que as do ciclo II, indicando um aquecimento gradual do protolito magmático com o decorrer do tempo (ou um aumento da temperatura dos processos magmatogênicos).

Os dois ciclos magmáticos mostram uma evolução regular em termos da sequência cronológica de suas unidades. Esta evolução é dada pela colocação sucessiva de magmas cada vez mais silicosos e claros (Fig. 4). O ciclo II inicia-se por uma unidade (CP) mais básica que a unidade inicial (BA) do ciclo I. Mas as rochas mais básicas do complexo são representadas pêlos diques de mela-quartzo monzonitos (DMP/DME) que não puderam ser caracterizados como mais um ciclo magmático pelo número insuficiente de dados disponíveis.

A posição dos turmalina-muscovita aplitos (APL), em relação aos dois ciclos magmático é ainda duvidosa, quer sobre os elementos maiores quer em termos de seus ETR (Wernick et al 1992), decorrente de sua mineralogia con- trastante em relação às demais unidades magmáticas. Dados geológicos indicam uma maior vinculação com o ciclo I (ver Tab. 1, coluna "Frequência de aplitos"), relação também sugerida por alguns dos diagramas geoquímicos. Fenómenos de turmalinização afetam em maior ou menor grau as fases magmáticas finais de todos os complexos granitóides intrusivos no Grupo São Roque independentemente de sua classificação serial (Artur et al. 1992) e atingem seu nível máximo no granito turmalinífero de Perus (Hasui 1963, Wernick 1983, Wernick et al. 1985, Dantas 1990), situado nas proximidades do complexo Itaqui.

Baseados em elementos maiores, principalmente $\mathrm{K}_{2} \mathrm{O}$ e $\mathrm{Na}_{2} \mathrm{O}$, cada um dos ciclos pode ser subdividido em dois subciclos (Fig. 4). O ciclo I compreende os subciclos IA (Unidade Bameri) e IB (Unidades Pedreira Cantareira e Torre) e o ciclo II os subciclos IIA (Unidades Cruz Preta e Aldeia da Serra) e IIB (Unidades Mutinga e Suni). A validade destas subdivisões é ressaltada nos diagramas geoquímicos que serão discutidos a seguir. 
Tabela 1 - Dados texturais e mineralógicos das unidades magmáticas do Complexo Itaqui. Convenções: tr - traços; **** muito frequentes; ***-frequentes; **-pouco frequentes; * - raros. Modificado de Ferreira (1991) e Ferreira \& Wernick (1991)

Table 1- Mineralogical and textural data for the igneous units of Itaqui Coraplex. Key: tr - trace; **** - very frequent; *** - frequent; ** - unusual; * - rare. After Ferreira (1991) and Ferreira \& Wernick (1991)

\begin{tabular}{|c|c|c|c|c|c|}
\hline \multirow{2}{*}{$\begin{array}{l}\text { UNIDADE } \\
\text { IGNEA }\end{array}$} & \multirow{2}{*}{$\begin{array}{c}\text { TEXTURA } \\
\text { CARACTERÍSTICAS }\end{array}$} & \multicolumn{2}{|c|}{ MEGACRISTAIS DE FK } & \multirow{2}{*}{$\begin{array}{l}\text { MINERAIS MÁFICOS } \\
\text { CARACTERÍSTICOS }\end{array}$} & \multirow{2}{*}{$\begin{array}{c}\text { FREQUENCIA } \\
\text { DE APLITOS }\end{array}$} \\
\hline & & $\%$ & DIMENSÃO MÉDIA & & \\
\hline BARUERI & \multirow{3}{*}{ Porfirbide } & 12 & $2,5 \mathrm{~cm}$ & \multirow{3}{*}{ Biotita e hornblenda } & Alta \\
\hline PEDREIRA & & 3 & $3 \mathrm{~cm}$ & & \multirow{3}{*}{ Média } \\
\hline CANTAREIRA & & & & & \\
\hline TORRE & Equigranular & & - & Biotita & \\
\hline DMP & Porfiritica & $0-3$ & $2,5 \mathrm{~cm}$ & Biotita e hornblenda & \multirow{3}{*}{ Média } \\
\hline DME & Equigranular & & - & Biotita e hornblenda . & \\
\hline CRUZ PRETA & Porfirítica & 13 & $1 \mathrm{~cm}$ & \multirow{5}{*}{ Biotita } & \\
\hline ALDEIA DA & \multirow{3}{*}{$\begin{array}{c}\text { Inequigranular } \\
\text { serial a } \\
\text { porfirítica }\end{array}$} & 3 & $1,5 \mathrm{~cm}$ & & Muito baixa \\
\hline SERRA & & & & & \\
\hline MUTINGA & & tr & $1,5 \mathrm{~cm}$ & & Nula \\
\hline SURU & Equigranular & \multirow{2}{*}{\multicolumn{2}{|c|}{-}} & & Nula \\
\hline APLITOS & Aplítica & & & Turmalina & - \\
\hline
\end{tabular}

\begin{tabular}{|c|c|c|c|c|c|c|c|c|c|c|}
\hline $\begin{array}{l}\text { UNIDADE } \\
\text { IGNEA }\end{array}$ & $\begin{array}{c}\text { BARUE- } \\
\text { RI }\end{array}$ & $\begin{array}{c}\text { PEDREIRA } \\
\text { CANTAREIRA }\end{array}$ & TORRE & DMP & DME & $\begin{array}{l}\text { CRUZ } \\
\text { PRETA }\end{array}$ & MUTINGA & $\begin{array}{l}\text { ALDELA } \\
\text { DA } \\
\text { SERRA }\end{array}$ & SURU & APLITOS \\
\hline PLAGIOGLÁSIO & 41 & 40 & 37 & 35 & 33 & 45 & 43 & 37 & 32 & 33 \\
\hline FELDSPATO-K & 17 & 32 & 33 & 22 & 33 & 26 & 27 & 28 & 39 & 34 \\
\hline QUARTZO & 24 & 22 & 24 & 7 & 14 & 16 & 23 & 24 & 27 & 29 \\
\hline BIOTTTA & 13 & 6 & 4 & 25 & 15 & 11 & 7 & 9 & 2 & $*$ \\
\hline HORBLENDA & 3 & 0,4 & - & 4 & 0,5 & - & & - & - & - \\
\hline TITANITA & 1 & 0,2 & 1 & 4 & 3 & 1 & 0,2 & 1 & $*$ & - \\
\hline OPACOS & 0,5 & 0,3 & 0,8 & 2 & 1,5 & 1 & * & 0,5 & $*$ & - \\
\hline APATITA & 0,5 & 0,1 & 0,2 & 1 & 0,3 & 0,5 & $*$ & 0,3 & $*$ & $\star$ \\
\hline ALLANITA & ***** & $* *$ & ** & - & $* *$ & $* *$ & $* * *$ & $* * * *$ & 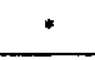 & $*$ \\
\hline ZIRCÃO & ** & $* *$ & $*$ & $*$ & * & * & $\overrightarrow{* *}$ & * & . & * \\
\hline EPIDOTO & $* *$ & $* *$ & - & ** & $* *$ & $* *$ & * & $*$ & - & - \\
\hline MUSCOVITA & - & - & - & - & - & - & - & - & - & 4 \\
\hline TURMALINA & - & - & - & - & - & - & - & - & - & $* *$ \\
\hline RUTILO & - & - & - & - & - & - & * & - & - & - \\
\hline
\end{tabular}

Evolução magmática A evolução magmática dos dois ciclos foi abordada em termos dos parâmetros catiônicos $(\mathrm{Na}+\mathrm{K}), \mathrm{Q}=\mathrm{Si} / 3-(\mathrm{K}+\mathrm{Na}+2 \mathrm{Ca} / 3), \mathrm{B}=(\mathrm{Fe}+\mathrm{Mg}+$ Ti), $P=K-(\mathrm{Na}+\mathrm{Ca}), \mathrm{R}=4 \mathrm{Si}-11(\mathrm{Na}+\mathrm{K})-2(\mathrm{Fe}-\mathrm{Ti}), \mathrm{R}_{2}=$ $(6 \mathrm{Ca}+2 \mathrm{Mg}+\mathrm{Al}),(\mathrm{Al}+\mathrm{Mg})-(\mathrm{K}+\mathrm{Fe}+2 \mathrm{Na}) \mathrm{e}(\mathrm{Al}+\mathrm{Fe})-$ $(\mathrm{K}+\mathrm{Mg}+4 \mathrm{Ca})$ desenvolvidos por De Ia Roche (1978) e aplicados entre outros por De Ia Roche et al. (1980), Debon (1980), Debon et al. (1988) e Batchelor \& Bowden (1985).

Os diagramas constam das figuras 5 (A, B, C), 6 e 7, nas quais os dados foram representados simultaneamente ora em sua totalidade ora pelas médias das diferentes unidades. Para a sua elaboração as unidades foram reunidas em cinco associações: 1. Barueri - Pedreira Cantareira - Torre (ciclo I); 2. diques máficos porfirítico e eqüigranular; 3 . Cruz Preta - Aldeia da Serra (subciclo IIA); 4. Mutinga-Suru (subciclo IIB); e 5. turmalina-muscovita aplitos.

Diagramas relacionando parâmetros catiônicos têm principalmente as seguintes vantagens:

- são capazes de distinguir rochas quimicamente muito semelhantes, como exemplificado pelo sistema de classificação $\mathrm{R}_{1} \times \mathrm{R}_{2}$;

- relacionam acuradamente o quimismo com a mineralogia das rochas tanto no caso de rochas em geral (De Ia Roche 1978, De Ia Roche et al. 1980) como para rochas granitóides em particular (Debon et al. 1988); 


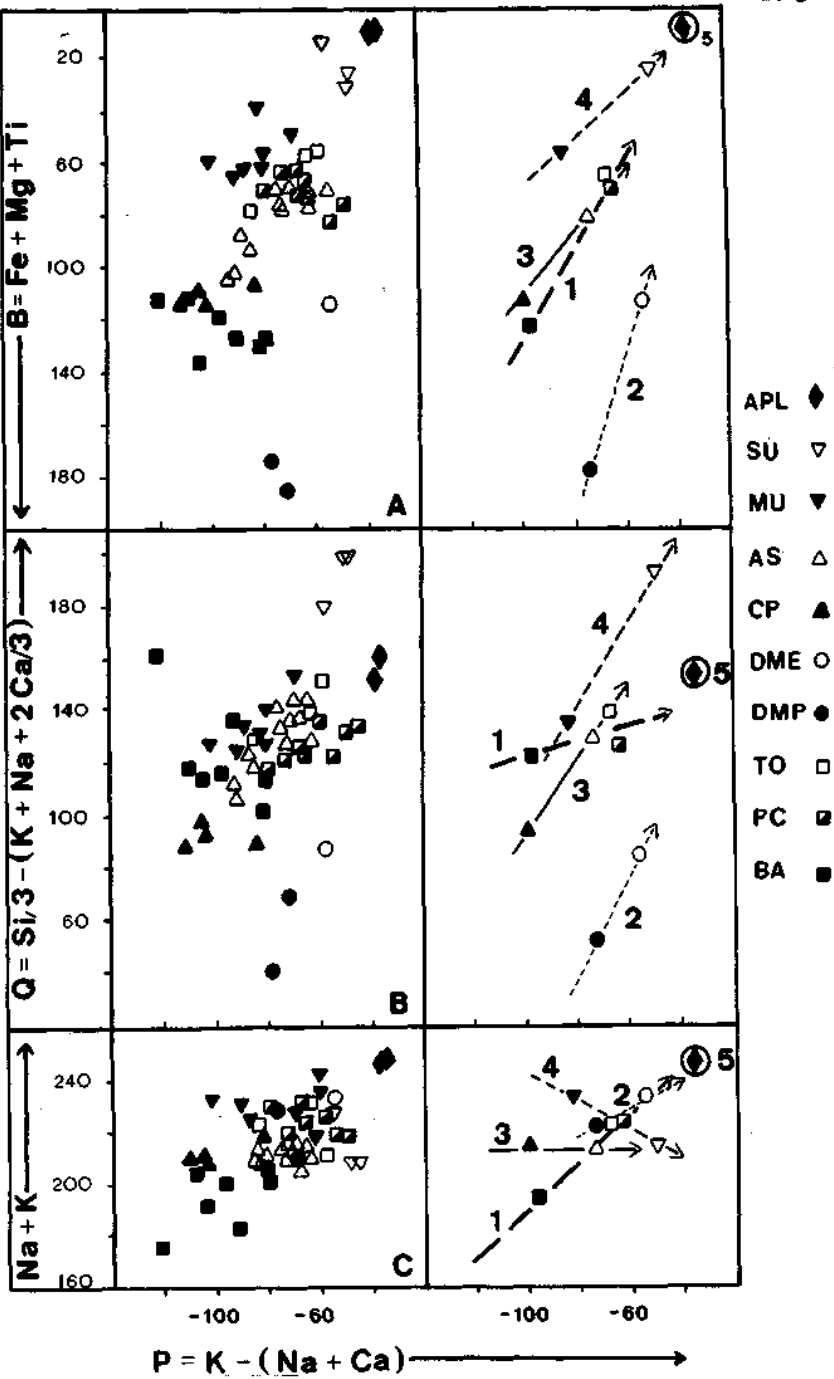

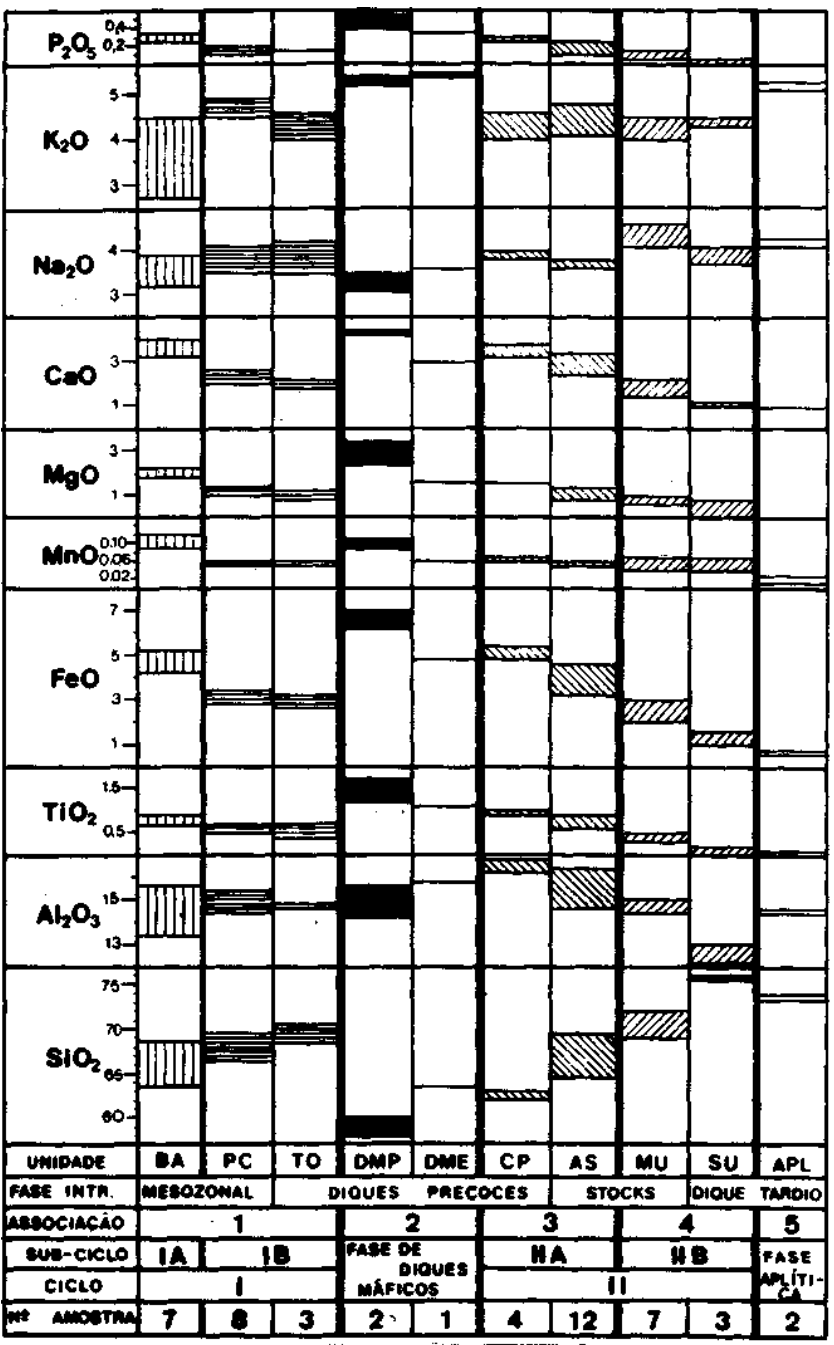

Figura 4 - Variação da composição química (em porcentagem de peso) das unidades magmáticas do Complexo Granitóide Itaqui. Símbolos conforme a figura 2. Segundo Wernick et al. (1993)

Figura 4 - Compositional variation (weight percent) of the igneous units of the Itaqui Complex. Symbols as in figure 2. After Wemick et al. (1993)

- permitem uma rápida compreensão do significado mineralógico de tendências evolutivas químicas (p. ex., Batchelor e Bowden 1985);

- não apresentam as distorções das tendências evolutivas que ocorrem em diagramas baseados em $\%$ de peso (Pearce 1969, Whitten 1975).

Diagrama $P x$ (Figura 5A) Este diagrama correlaciona o conteúdo em feldspato potássico (parâmetro $\mathrm{P}$ ) com o índice de coloração (parâmetro B). A figura 5A mostra uma correlação inversa destes parâmetros para as associações 1-4, suas disposições subparalelas e a convergência das tendências evolutivas rumo à associação 5.

A dispersão dos dados individuais da associação 1 (BA + PC + TO) indica claramente que a colocação das unidades magmáticas do ciclo I reflete saltos composicionais na fonte magmatogênica (definindo os subciclos IA e IB) e que cada unidade sofreu diferenciação in situ. $\mathrm{O}$ diagrama define claramente os sub-ciclos IIA e IIB (associaçōes 3 e 4), de disposição subparalela.

igura 5 - Distribuição das unidades ígneas do Complexo Itaqui nos diagramas multicationicos $P$ versus $B(5 A), Q$ (5B) e $\mathrm{Na}+K(5 \mathrm{C})$. A esquerda estão representados os dados totais e a direita as médias por unidade magmática e suas tendências evolutivas. Símbolos segundo afigura 2 Figure 5 - Distribution of igneous units of Itaqui complex in the multicationic diagrams $\mathrm{P}$ versus $\mathrm{B}(5 \mathrm{~A}), \mathrm{Q}(5 \mathrm{~B})$ and $\mathrm{Na}+\mathrm{K}(5 \mathrm{C})$. Ali data on the left, averages for each unit on the right with their evolutionary trends. Key as figure 2

Diagrama PxO (Fig. 5B) Este diagrama correlaciona o conteúdo em feldspato potássico (índice $\mathrm{P}$ ) com o conteúdo em quartzo (índice $\mathrm{Q}$ ). Neste gráfico o ciclo I distingue-se claramente dos subciclos IIA e IIB (de disposição subparalela) por um ganho mais rápido no teor de feldspato potássico em relação ao incremento no teor de quartzo. Dada a natureza porfiróide das unidades BA e PC, este aspecto parece indicar a concentração de feldspato potássico pela aglutinação de megacristais por fluxo magmático e/ou filtragem sob compressão. As numerosas concentrações de megacristais de microclina com formas alongadas, irregulares e tabulares (ver figuras 16 e 20 em Ferreira 1991), que ocorrem na unidade Barueri sustentam esta interpretação.

O diagrama sugere ainda uma vinculação entre o ciclo I (associação 1) e os aplitos (associação 5) e destaca-se novamente o paralelismo das tendências evolutivas dos subciclos IIA (associação 3) e IIB (associação 4).

Diagrama $P \times(\mathrm{Na}+\mathrm{K})$ (Figura 5C) Trata-se de um diagrama complementar ao anterior, colocando em evidência a alcalinidade das rochas (parâmetro $\mathrm{Na}+\mathrm{K}$ ) na depen- 
dência do seu teor em feldspato potássico. Aqui, é interessante ressaltar o comportamento diferencial das associações 1, 3 e 4, nas quais, respectivamente, o teor de álcalis cresce, permanece constante e decresce com o aumento do índice P. $\mathrm{O}$ diagrama sugere, à semelhança do diagrama $\mathrm{P} \times \mathrm{Q}$, uma possível vinculação genética entre as rochas do ciclo I com os aplitos da associação 5. Finalmente, como todos os diagramas precedentes, aponta à convergência composicional entre as unidades magmáticas terminais do ciclo I (Unidades Pedreira Cantareira e Torre) e do subciclo IIA (unidade Aldeia da Serra).

Diagrama $R 1 \times 1 \quad R 2$ (Figura 6) Este diagrama correlaciona, em rochas granitóides, basicamente quatro aspectos da evolução magmática, das quais três têm o sentido de sua variação assinalados na figura 6: 1. teor em anortita no plagioclásio; 2. teor em quartzo; 3. relação feldspato alcalino/plagioclásio; e 4 . a cristalização sucessiva de clinopiroxênios, anfibólios e biotitas.

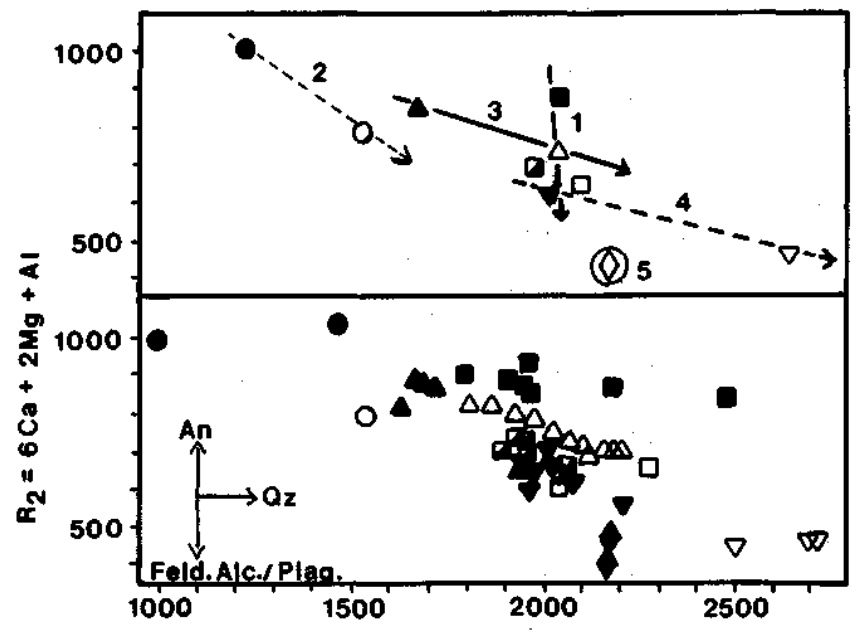

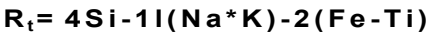

Figura 6 - Distribuição das unidades ígneas do Complexo Itaqui no diagrama multicationico $R_{t} R_{2}$. Dados totais na parte inferior e médias por unidades e suas tendências evolutivas na parte superior. Símbolos segundo afigura 2 Figure 6 - Distribution of igneous units of Itaqui complex in the multicationic diagram $\mathrm{R}, \mathrm{R}_{2}$. Ali data below, averages for each unit and their evolutionary trends above. Key as figure 2

Ás diferenças nas tendências evolutivas médias globais entre as associações 1, 3 e 4 confirmam as evoluções parciais discutidas nas figuras $5 \mathrm{~A}, 5 \mathrm{~B}$ e $5 \mathrm{C}$, destacando a dife-rença entre os ciclos I e II, o paralelismo evolutivo dos subciclos IIA e IIB e a vinculação genética dos aplitos com o ciclo L

Finalmente, sobre as amostras individuais, destacam-se nítidas diferenças evolutivas quando são comparadas unidades quimicamente expandidas do ciclo I (Unidade Barueri) com $\mathrm{R}_{2}$ aproximadamente constante e do ciclo II (Unidade Aldeia da Serra) com $R_{2}$ decrescente.

Diagrama $\mathrm{Al}+\mathrm{Mg}-(\mathrm{K}+\mathrm{Fe}+2 \mathrm{Na}) \mathrm{xAl}+\mathrm{Fe}-(\mathrm{K}+\mathrm{Mg}+$ $4 \mathrm{Ca}$ ) (Figura 7) Este diagrama representa a função do plano $2 \mathrm{~F}=\mathrm{x}+\mathrm{y}$ no sistema $\mathrm{xy}$, onde $\mathrm{x}=(\mathrm{Al}-\mathrm{K})-(\mathrm{Fe}-\mathrm{Mg})$ $-2 \mathrm{Na}$ e $\mathrm{y}=(\mathrm{Al}-\mathrm{K})+(\mathrm{Fe}-\mathrm{Mg})-4 \mathrm{Ca}$ (De Ia Roche, 1978). Quando $2 \mathrm{~F}=\mathrm{O}$, o plano contém os principais minerais das rochas graníticas: quartzo, albita, anortita, feldspato potássico e biotita. Rochas situadas acima deste plano (cuja projeção no diagrama é dada pela diagonal da Fig. 7) são peraluminosas e as situadas abaixo tendem a ser portadoras de hornblenda (rochas metaluminosas). O diagrama reflete as variações nos conteúdos de $\mathrm{Ga}$ e $\mathrm{Na}$ e das relações

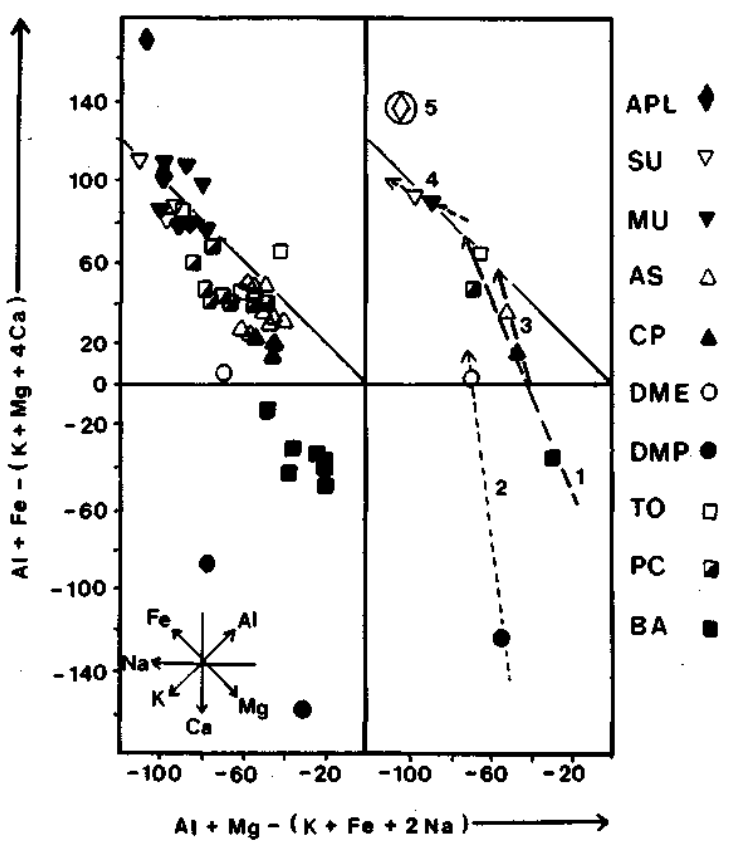

Figura 7 - Distribuição das unidades ígneas do Complexo Itaqui no diagrama multicatiônico $\mathrm{Al}+\mathrm{Mg}-(\mathrm{K}+\mathrm{Fe}+$ 2Na) $x \mathrm{Al}+\mathrm{Fe}-(\mathrm{K}+\mathrm{Mg}+4 \mathrm{Ca})$. A esquerda estão representados os dados totais e a direita as médias por unidade magmática e suas tendências evolutivas. Símbolos segundo afigura 2

Figure 7 - Distribution of igneous units of Itaqui complex in the multicationic diagram $\mathrm{Al}+\mathrm{Mg}-(\mathrm{K}+\mathrm{Fe}+2 \mathrm{Na}) \times \mathrm{Al}+\mathrm{Fe}-(\mathrm{K}+\mathrm{Mg}+$ $4 \mathrm{Ca}$ ). Ali data on the left, averages for each unit on the right with their evolutionary trends. Key as figure 2

$\mathrm{Fe} / \mathrm{Mg}$ e $\mathrm{Al} / \mathrm{K}$. Com exceção da associação 5 (rochas com turmalina e muscovita), as demais situam-se sobre ou abaixo do plano $2 \mathrm{~F}=0$.

A diminuição da relação hornblenda/biotita na Unidade Pedreira Cantareira (PC) em relação à Unidade Barueri (BA) e a presença apenas de biotita na Unidade Torre (TO), que caracteriza a mineralogia fêmica do ciclo I, acha-se muito bem definida na figura 7 , refletindo que seu processo evolutivo é acompanhado por um aumento das relações Fe/ $\mathrm{Mg}, \mathrm{Al} / \mathrm{K}$ e no teor em $\mathrm{Na}$ e por perdas no teor em Ca (o que implica em plagioclásios cada vez mais sódicos). Já, a associação 4 , contendo apenas biotita, evolui sobre o plano $2 \mathrm{~F}=$ $\mathrm{O}$ pelo fracionamento de biotita e plagioclásio.

A evolução do subciclo IIA é semelhante a do ciclo I resultando na já mencionada convergência composicional entre as Unidades Pedreira Cantareira, Torre e Aldeia da Serra (o que pode sugerir a ocorrência esporádica de hornblenda na Unidade Cruz Preta). Finalmente, a figura 7 sugere novamente a vinculação entre os aplitos e a evolução do ciclo I.

CONCLUSÕES 1. O complexo granitóide cálcio-alcalino Itaqui, associado à Falha Transcorrente Taxaquara, é constituído por dez unidades magmáticas reunidas em quatro episódios de acresção, que se relacionam com fases de removimentação da falha que num dos seus estágios finais de evolução secciona o complexo no seu sentido longi-tudinal. 2. As dez unidades são representadas essencialmente por granodioritos e monzogranitos com textura porfiróide, porfirítica, inequigranular e equigranular com variável índice de coloração e contendo tanto hornblenda e biotita quanto apenas biotita. Os aplitos de colocação final são portadores de turmalina e muscovita. 
3. Sete unidades foram reunidas em dois ciclos magmáticos maiores. O ciclo I compreende as Unidades Barueri, Pedreira Cantareira e Torre e o ciclo n as Unidades Cruz Preta, Aldeia da Serra, Mutinga e Suru. Caracterís-ticas comuns aos dois ciclos são a seqüenciaçãp temporal das unidades de um pólo mais básico para um mais ácido e a variação textural na qual as unidades iniciais são porfiróides ou porfiríticas e as finais equigranulares. Entretanto, os dois ciclos distinguem-se sob o aspecto mineralógico qualitativo e quantitativo e ao nível de seus elementos quími-cos maiores. Destaca-se, ainda, que o ciclo $n$ inicia-se com um magmatismo mais básico que o magmatismo inicial do ciclo $\mathrm{L}$

4. Os dois ciclos são separados por uma fase de colocação de diques de mela-quartzo monzonitos reunidos em duas unidades magmáticas. Não existem dados químicos su-ficientes para caracterizá-los como um ciclo magmático independente, mas os dados preliminares apontam nessa direção.

5. A última manifestação magmática do complexo é dada por turmalina-muscovita aplitos. Geograficamente, a sua ocorrência coincide essencialmente com a área de exposição das rochas do ciclo I. Vários diagramas geoquímicos sugerem sua vinculação com esse ciclo, apesar de sua mineralogia anómala em termos dos minerais fêmicos relacionados às demais unidades do complexo.

6. Cada ciclo pode ser subdividido em dois subciclos, tendo por base vários critérios químicos.

7. Cada ciclo (ou subciclo) mostra tendência evolutiva distinta, ressaltada em diversos diagramas catiônicos. Entretanto, na maioria dos casos, estes assinalam a mesma tendência para os subciclos HA e UB. A evolução faz-se por uma combinação de fracionamento de minerais máficos, plagioclásio e acessórios. Na Unidade Barueri, este processo é sustentado pela presença de enclaves cognatos muito enriquecidos em minerais fếmicos e acessórios. Adicionalmente, para o ciclo I, os dados químicos são sugestivos para a operação de processos de fracionamento por fluxo magmático e/ou filtragem por compressão. 8. A caracterização de ciclos e subciclos magmáticos no Granito Itaqui implica na sua definição como complexo múltiplo e composto.

9.O término dos subciclos BB, HA E UB transgride o limite da fase intrusiva na qual se iniciaram. $O$ subciclo IB inicia-se na fase intrusiva mesozonal (Unidade Pedreira Cantareira) e termina na fase de colocação dos diques precoces (Unidade Torre). O subciclo HA inicia-se nesta fase (Unidade Cruz Preta) e termina na fase de colocação dos stocks (Unidade Aldeia da Serra). O subciclo HB inicia-se nesta fase (Unidade Mutinga) e termina na fase intrusiva de colocação dos diques tardios (Unidade Suru). Esse aspecto indica a existência de uma defasagem temporal entre as mudanças nas condições tectônicas e as mudanças nas condições magmatogênicas refletidas pela geração de um novo subciclo magmático.

Agradecimentos $\mathrm{O}$ autor E.W. agradece ao CNPq (processo 500459/90-8) e à FINEP (processo 4.3.90.0315.00), e o autor CJ.F. à FAPESP (processo 92/1333-5) pelo suporte dado à realização deste trabalho.

\section{REFERÊNCIAS BIBLIOGRÁFICAS}

ARTUR, A C - WERNICK, E - ANDRADE, FR D 1992. Turmalina granitos dos Grupos São Roque e Açungui, Estado de São Paulo. In: CONGR. BRAS. GEOL., 37. São Paulo, 1992. Boletim de Resumos Expandidos... São Paulo, SBG. v. 1, p. 354-355.

BATCHELOR, R.A. \& BOWDEN, P. 1985. Petrogenetic interpretation of granitoid rock series using multicationic parameters. Chem. Geol, 48:43-55.

BISTRICHI, C.A.; CARNEIRO, C.D.R.; DANTAS, A.S.L.; PONCANO, W.L.; CAMPANHA, G.A.C.; NAGATA, N.; ALMEIDA, M.A.; STEIN, D. P.; MELO, M.S.; CREMONINI, O.A. 1981. Mapa geológico do Estado de São Paulo, 1:500.000. São Paulo, IPT.

DANTAS, A.S.L. 1990. Geologia da Faixa São Roque e Intrusivas Associadas na Região Entre São Paulo e Mairiporã, Norte de São Paulo, SP. São Paulo. 199 p. (Dissertação de Mestrado, IG-USP).

DEBON, F. 1980. Génesis of three concentrically-zoned granitoid Plutons of Cauterets-Panticosa (French and Spanish Western Pyrenees). Geol. Rundsch., 69:107-130.

DEBON, F.; L̂E FORT, P.; SABATÉ, P. 1988. Uma classificação químicomineralógica das rochas plutônicas comuns e suas associações, método e aplicacões. Rev. Bros. Geoc., 18(2):122-133.

DE LA ROCHE, H. 1978. La chimie dês roches présentée et interpretée d'aprés Ia structure de leur fácies mineral dans l'espace dês variables chimiques: fonctions spécifiques et diagrammes qui s'en déduisent application aux roches ignées. Chem. Geol. 21:63-87.

DE LA ROCHE, H.; LETERRIER, J; GRANDCLAUDE, P; MARCHAL, M. 1980. A classification of volcanic and plutonic rocks using RjRjdiagram and major-element analyses - its relationships with currrent nomenclature. Chem. Geol., 29:183-210.

FERREIRA, C.J. 1991. Geologia, Petrografia e Topologia de Zircão da Suite Intrusiva Itaqui, Barueri (SP). Rio Claro. 253 p. (Dissertação de Mestrado, IGCE-UNESP)

FERREIRA, C J. \& WERNICK, E. 1989. Granito Itaqui (SP): caracterizacão das fácies e relações temporais. In: SIMP. REG. GEOL., 1. Rio de Janeiro, 1989. Atas... Rio de Janeiro, SBG. p. 171-172

FERREIRA, C.J. \& WERNICK, E. 1991. Petrografia da Suíte Intrusiva Itaqui (SP). In: SIMP. REG. GEOL., 2. São Paulo, 1991. Atas... São Paulo, SBG. p. 121-130.

FERREIRA C.J.; WERNICK, E. HÕRMANN, P.K. 1992. Evolucão magmática e tipologia de zircão no Complexo Granitóide Itaqui, SP. In: CONGR. BRÁS. GEOL., 37. São Paulo, 1992. Boletim de Resumos Expandidos... São Paulo, SBG. v. 1, p. 330-331.

HASUI, Y. 1963. Sobre os granitos turmaliníferos de Perus, São Paulo (SP) Boi Soe Brás Geol, 12·37-108.

JANASI, VA. \& ULBRICH, H.H.G.J. 1992. Inventário bibliográfico de granitos do Estado de São Paulo. Boi. IG-USP, Publ. Esp., 11:1-253.

JANASI, V.A.; VASCONCELLOS, A.C.B.C.; VLACH, S.R.F.; MOTIDOME, MJ. 1990. Granitóides da região entre as cidades de São
Paulo e Piedade (SP): faciologia e contexto tectônico. In: CONGR. BRÁS. GEOL., 36. Natal, 1990. Anais... SBG, Natal. 4:1925-1935.

PEARCE, T.H. 1969. A contribution to the theory of variation diagrams. Contrib. Mineral Petrol, 19:142-157.

PHILPOTTS, A.R. 1990. Principies of Igneous and Metamorphic Petrology. New Jersey, Prentice Hall, Englewood Cliffs. 498 p.

PITCHER, W.S. 1985. A multiple and composite batholith. In: PITCHER, W.S.; ATHERTON, M.P.; COBBING, E.J. eds. Magmaiism at a Plaie Edge. New York, Wiley \& Sons. p. 93-101.

WERNICK, E. 1983. Granitos na regiấo da Grande São Paulo. In: SIMP. REG. GEOL., 4. São Paulo, 1983. Guia de Excursão... São Paulo, SBG. $21 \mathrm{p}$

WERNICK, E. \& FERREIRA, C.J. 1987. Um expressivo dique sinintrusivo no Granito Itaqui, arredores de São Paulo. In: SIMP. REG. GEOL., 6. Rio Claro, 1987. Atas... São Paulo, SBG. v. 1, p. 135-148.

WERNICK, E. \& FERREIRA, C.J. 1993. Estrutura, arquitetura e evolução do Complexo Granitóide Itaqui, SP. Geociências, 12(1):89-109.

WERNICK, E.; HORMANN, P.K.; FERREIRA, C.J. 1991. Processos de evolucão magmática no Complexo Granitóide Itaqui, SP, Brasil. In: CONGR. BRAS. GEOQ., 3, e CONGR. GEOQ. DOS PAISES DE LÍNGUA PORTUGUESA, 1. São Paulo, 1991. Boletim de Resumos... São Paulo, SBGq. p. 271-275.

WERNICK, E.; HORMANN, P.K.; FERREIRA, C.J. 1992. Viscosidade e evolução de magmas granitóides cálcio-alcalinos: uma abordagem através de ETR. Rev. Brás. Geoç., 23(2):

WERNICK, E.; FERREIRA, C.J.; HÔRMANN, P.K. 1993. Evolução das unidades magmáticas do Complexo Granitóide Itaqui (Pré-Cambriano Superior), Estado de São Paulo, Brasil: aspectos geológicos, petrográficos e geoquímicos. In: SIMP. INTERNACIONAL DEL NEOPROTEROZOICO - CAMBRICO DE LA CUENCA DEL PLATA, 1. Uruguai, 1993. Boletim de Resumos... Montevideo, DI.NA.MI.GE. 6

WERNICK, E.; WEBER-DIENFENBACH, K; CORREIA, P.R.; CERQUEIRA, L.C.C. 1985. Os Granitos Mairiporã, Cantareira e Perus, arredores de São Paulo: dados químicos, tipologia de zircão e uma interpretação preliminar. In: SIMP. REG. GEOL., 5. São Paulo, 1985. Atas.. São Paulo, SBG. v. 1, p. 3-18.

WHITTEN, E.H.T. 1975. Appropriate units for expressing Chemical composition of igneous rocks. In: WHITTEN, E.H.T. ed Quantitative Studies in the Geological Science. Geol. Soe. Am. Mem., 142:283-308.

Manuscrito A785

Recebido em 31 de agosto de 1993

Revisão do autor em 23 de fevereiro de 1994 Revisão aceita em 23 de fevereiro de 1994 